\title{
Cut Mellin moments approach and generalized DGLAP equations
}

\section{Dorota Strózik-Kotlorz ${ }^{* \dagger}$}

Opole University of Technology, Poland

E-mail: dorota@theor.jinr.ru

\section{S. V. Mikhailov}

Bogoliubov Laboratory of Theoretical Physics, JINR, 141980 Dubna, Russia

E-mail: mikhs@theor.jinr.ru

\section{O. V. Teryaev}

Bogoliubov Laboratory of Theoretical Physics, JINR, 141980 Dubna, Russia

E-mail: teryaev@theor.jinr.ru

\begin{abstract}
Recent results on the generalization of the DGLAP evolution equations within the cut Mellin moments (CMM) approach are presented. The generalized CMM obtained by multiple integrations as well as multiple differentiations of the original parton distribution also satisfy the DGLAP equations with the simply transformed evolution kernel. A similar generalized evolution equation, with the correspondingly modified coefficient functions, can also be obtained for structure functions. We present classes of CMM and corresponding to them DGLAP evolution kernels. We give an example of application of the CMM approach to analysis of the experimental data on the Bjorken sum rule. Using appropriate classes of CMM for the available experimental kinematic range enables enhancement of the $x$-region with smaller uncertainties in the analysis.
\end{abstract}

XXII International Baldin Seminar on High Energy Physics Problems

15-20 September, 2014

JINR, Dubna, Russia

\footnotetext{
*Speaker.

$\dagger$ This work is supported by the Bogoliubov-Infeld Program, Grant No 01-3-1113-2014/2018.

${ }^{\ddagger}$ Support from the Russian Foundation for Fundamental Research (Grant No. 14-01-00647a).
} 


\section{Introduction}

A central role in QCD analysis of high energy processes is usually played by the parton distribution functions (pdf) $f\left(x, Q^{2}\right)$ providing information about the hadron structure and the strong interaction. The scale $Q^{2}$-evolution of pdf, one of the most important predictions of perturbative QCD is governed by the well-known DGLAP evolution equations [1-4]. Alternatively, one can study how to evolve with the scale $Q^{2}$ the Mellin moments of the parton density $f\left(n, Q^{2}\right)$, which are integrals of pdf weighted by $x^{n}$ over the whole range of $x, 0 \leq x<1$. These moments provide a natural framework of QCD analysis as they originate from the basic formalism of operator product expansion (OPE). Simultaneously, however, the moments are some sort of idealization and it is useful to invent new "real" observables, named the "cut (truncated) Mellin moments" (CMM) with a goal to overcome kinematic constraints naturally appearing in real DIS and hadron-hadron collisions. The CMM $f\left(z, n, Q^{2}\right)$ are generalized moments of the parton density $f\left(x, Q^{2}\right)$ with the lower limit of integration $z \equiv x_{\min }>0$ and in this way the kinematic constraint is taken into account. Namely, arbitrarily small values of the variable $x$ cannot be reached in experiments.

The idea of "truncated" Mellin moments of the parton densities in QCD analysis was introduced and developed in the late 1990's [5-8]. The authors obtained the nondiagonal differential evolution equations, in which the $n$th truncated moment couples to all higher ones. Later on, diagonal integro-differential DGLAP-type evolution equations for the single and double truncated moments of the parton densities were derived in [9] and [10,11], respectively. The main finding of the truncated Mellin moments approach (CMMA) is that the $n$th moment of the parton density obeys the DGLAP equation again, but with a rescaled evolution kernel $P^{\prime}(z)=z^{n} P(z)$ [9]. The CMMA has already been successfully applied, e.g., in spin physics to derive a generalization of the Wandzura-Wilczek relation in terms of the truncated moments and to obtain the evolution equation for the structure function, e.g., $g_{2}[11,12]$. Truncation of the moments in the upper limit is less important in comparison with the low- $x$ limit because of the rapid decrease of the parton densities as $x \rightarrow 1$; nevertheless, a comprehensive theoretical analysis requires an equal treatment of both truncated limits. The evolution equations for double cut moments and their application to study the quark-hadron duality were also discussed in [13]. Quite recently a valuable generalization of the CMM approach incorporating multiple integrations as well as multiple differentiations of the original parton distribution has been obtained [14]. This novel generalization of CMM and the corresponding DGLAP equations provides a powerful tool to test QCD at experimental constraints.

\section{Generalized DGLAP equations}

Here we present the main results on the novel general solutions for the DGLAP equations [14]. If $f\left(x, Q^{2}\right)$ is a solution of the DGLAP equation with the kernel $P(y)$ :

$$
\dot{f} \equiv \frac{\partial f\left(z, Q^{2}\right)}{\partial \ln Q^{2}}=(P * f)(z) \equiv \int_{0}^{1} P(y) f\left(x, Q^{2}\right) \delta(z-x y) d x d y,
$$


then the multi-integrated function which is a generalization of the CMM

$$
f\left(z ; n_{1}, n_{2}, \ldots, n_{k}\right)=\int_{z}^{1} z_{k}^{n_{k}-1} d z_{k} \int_{z_{k}}^{1} z_{k-1}^{n_{k-1}-1} d z_{k-1} \ldots \int_{z_{2}}^{1} z_{1}^{n_{1}-1} f\left(z_{1}\right) d z_{1}
$$

is also the solution of DGLAP equation:

$$
\dot{f}\left(z ; n_{1}, n_{2}, \ldots, n_{k}\right)=(\mathscr{P} * f)\left(z ; n_{1}, n_{2}, \ldots, n_{k}\right)
$$

with the kernel

$$
\mathscr{P}(y)=P(y) \cdot y^{n_{1}+n_{2}+\ldots+n_{k}} .
$$

The general solution (2.2) is the source of various new partial solutions and also already known results. Namely, for $n_{1}=n_{2}=\ldots=n_{k}=n$ one has

$$
\begin{aligned}
f\left(z ;\{n\}_{k}\right) & =\int_{z}^{1} z_{k}^{n-1} d z_{k} \int_{z_{k}}^{1} z_{k-1}^{n-1} d z_{k-1} \ldots \int_{z_{2}}^{1} z_{1}^{n-1} f\left(z_{1}\right) d z_{1} \\
& =\int_{z}^{1}\left[\frac{t^{n}-z^{n}}{n}\right]^{k-1} \frac{f(t)}{(k-1) !} t^{n-1} d t
\end{aligned}
$$

with the corresponding evolution kernel

$$
\mathscr{P}(y)=P(y) \cdot y^{k n} .
$$

For $k=1$ (2.5) reduces to the cut $n$-th moment

$$
f(z ; n)=\int_{z}^{1} t^{n-1} f(t) d t
$$

and one obtains the original evolution equation [9]:

$$
\dot{f}(z, n) \equiv \frac{\partial f\left(z ; n, Q^{2}\right)}{\partial \ln Q^{2}}=(\mathscr{P} * f)\left(z ; n, Q^{2}\right) \equiv \int_{0}^{1} \mathscr{P}(y) f\left(x ; n, Q^{2}\right) \delta(z-x y) d x d y,
$$

where

$$
\mathscr{P}(y)=P(y) \cdot y^{n} .
$$

If one puts $z=0$ in (2.8), it reduces to the well known standard renorm-group equation for the moments $f\left(0 ; n, Q^{2}\right)$ :

$$
\frac{\partial f\left(0 ; n, Q^{2}\right)}{\partial \ln Q^{2}}=\left(\int_{0}^{1} P(y) y^{n-1} d y\right) \cdot f\left(0 ; n, Q^{2}\right) \equiv \gamma(n) \cdot f\left(0 ; n, Q^{2}\right) .
$$

Consider another special case, where $n_{1}=n$ and $n_{2}=n_{3}=\ldots=n_{k}=0$. Then it can be easily shown that

$$
\begin{aligned}
f(z ; n, 0, \ldots, 0) & =\int_{z}^{1} \frac{d z_{k}}{z_{k}} \int_{z_{k}}^{1} \frac{d z_{k-1}}{z_{k-1}} \ldots \int_{z_{2}}^{1} z_{1}^{n} f\left(z_{1}\right) \frac{d z_{1}}{z_{1}} \\
& =\int_{z}^{1} \frac{\ln ^{(k-1)}(x / z)}{(k-1) !} x^{n-1} f(x) d x
\end{aligned}
$$


evolves with the kernel which is independent of $k$ :

$$
\mathscr{P}(y)=P(y) \cdot y^{n} .
$$

We discuss this special case and its implication for experimental data analysis in the next section. The CMM approach (2.2)-(2.4) can also be extended to negative values of $k$ providing, e.g., DGLAP-like equations for multiple differentiation of the original parton distribution. To this aim, we consider the next special case of (2.2), namely

$$
f(z ; 1,1, \ldots, 1)=\int_{z}^{1} d z_{k} \int_{z_{k}}^{1} d z_{k-1} \ldots \int_{z_{2}}^{1} f\left(z_{1}\right) d z_{1}=\int_{z}^{1}(x-z)^{k-1} \frac{f(x)}{(k-1) !} d x
$$

which, according to (2.4), evolves with the kernel

$$
\mathscr{P}(y)=P(y) \cdot y^{k}
$$

Since $f(z)$ can be expressed via an inverse operation on $f(z ; 1,1, \ldots, 1)$ :

$$
f(z)=\left(-\frac{d}{d z}\right)^{k} f(z ; 1,1, \ldots, 1)
$$

one gets immediately the generalized CMM approach extended to the multi-differentiation. Namely, on the basis of (2.1), (2.13), (2.14) and (2.15) one can find that $k$ th derivative of the solution $f$,

$$
(-1)^{k} f^{(k)}(z)=\left(-\frac{d}{d z}\right)^{k} f(z)
$$

obeys the DGLAP evolution with the corresponding kernel

$$
\mathscr{P}(y)=P(y) \cdot y^{-k}
$$

More generally, the derivative of the function $\varphi_{n}(z) \equiv z^{n} f(z)$

$$
(-1)^{k} \varphi_{n}^{(k)}(z)=\left(-\frac{d}{d z}\right)^{k}\left[z^{n} f(z)\right]
$$

has the evolution kernel

$$
\mathscr{P}(y)=P(y) \cdot y^{n-k} .
$$

Strictly speaking, in the CMM generalization integer $k>0$ corresponds to the multi-integration, while integer $k<0$ corresponds to the multi-differentiation of the initial function. The special solutions $\varphi_{1}^{(k)}(x)=(x f(x))^{(k)}$ were considered in $[15,16]$. It is worthy to notice that the CMM approach can be analytically extended to any real value of $k$. In this case, factors $(k-1)$ ! in all above formulas should be replaced by $\Gamma(k)$. In Table 1, we collect a few partial results for $\varphi_{n}^{(k)}$.

The generalized evolution equation similar to (2.2)-(2.3) can also be obtained for the structure function (SF) $\mathscr{F}$. If $F=C * f$ denotes the original SF and obeys the evolution equation [17]

$$
\dot{F}\left(z ; \mu^{2}\right)=(K * F)(z)
$$




\begin{tabular}{|c|c|c|c|c|}
\hline$k=2$ & $k=1$ & $k=0$ & $k=-1$ & $k=-2$ \\
\hline $\int_{x}^{1} d z \int_{z}^{1} \varphi_{n}(t) d t$ & $\int_{x}^{1} \varphi_{n}(t) d t$ & $\varphi_{n}(x)$ & $-\varphi_{n}^{\prime}(x)$ & $\varphi_{n}^{\prime \prime}(x)$ \\
$P(y) y^{n+2}$ & $P(y) y^{n+1}$ & $P(y) y^{n}$ & $P(y) y^{n-1}$ & $P(y) y^{n-2}$ \\
\hline
\end{tabular}

Table 1: Partial results of the CMM generalization of the DGLAP equations for $\varphi_{n}(z) \equiv z^{n} f(z)$.

where

$$
K=P+\beta\left(a_{s}\right)\left(\partial_{a_{s}} C\right) * C^{-1},
$$

and $\beta$ is the QCD $\beta$-function, then one can obtain the new SF $\mathscr{F}$ and the new coefficient function $\mathscr{C}$ [12], [14]:

$$
F, C \rightarrow \mathscr{F}=\mathscr{C} * f\left(z ;\{n\}_{k}\right), \mathscr{C}=C(t) \cdot t^{n_{1}+n_{2}+\ldots+n_{k}} .
$$

Finally, one arrives at the evolution equation for $\mathscr{F}$ :

$$
\dot{\mathscr{F}}\left(z ;\{n\}_{k}\right)=\mathscr{K} * \mathscr{F}\left(z ;\{n\}_{k}\right)
$$

with the kernel

$$
\mathscr{K}(y)=K(y) \cdot y^{n_{1}+n_{2}+\ldots+n_{k}} .
$$

\section{Applications to experimental data analysis}

Based on gCMMA different interesting partial solutions of the generalized DGLAP equations can be constructed and applied to analyse the experimental data in different restricted $x$-regions, respectively. In Table 2, we summarize our main results which can be useful for this purpose. In the first column, we present the generalized CMM (2.2) and in the second column, we present the corresponding DGLAP evolution kernels. As we have mentioned in the previous section, a special case of CMM, (2.11) (also fifth row in Table 2) would be appropriate for analysis of the experimental data, e.g., the DIS sum rules. One can see that the corresponding DGLAP kernel (2.12) is independent of $k$. Hence, integrands $\ln ^{(k-1)}(x / z) / \Gamma(k)$ at different $k$ are "bricks" for any new gCMM constructions that evolve following the same DGLAP equation. The contribution to $f(z ; n, 0, \ldots, 0)$ is reinforced at the right end $x=1$ by powers of logs. This reinforcement becomes especially useful for the case when the experimental data are better known at larger $x$ and, in contrast, ones are unreliable or worse known at lower $x$. In Fig. 1, we show contributions to the Bjorken Sum Rule $(n=0)$, coming from the restricted region $x_{0}<x<1$, generalized to the form

$$
\int_{x_{0}}^{1} \frac{\ln ^{(k-1)}\left(x / x_{0}\right)}{\Gamma(k)} g_{1}^{N S}(x) \frac{d x}{x},
$$

which allows one to enhance the larger- $x$ region stronger with growing $k$. Taking into account that for $0<x \leq 1$

$$
x=\sum_{k=0}^{\infty} \frac{\ln ^{k} x}{k !},
$$




\begin{tabular}{|c|c|c|}
\hline 1. & Generalized CMM & DGLAP kernel \\
\hline 2. & $f(x)$ & $P(y)$ \\
3. & $x^{n} f(x)$ & $P(y) \cdot y^{n}$ \\
4. & $\int_{z}^{1} d x x^{n-1} f(x)$ & $P(y) \cdot y^{n}$ \\
5. & $f(z ; n, 0, \ldots, 0)=\int_{z}^{1} \frac{\ln ^{(k-1)}(x / z)}{(k-1) !} x^{n-1} f(x) d x$ & $P(y) \cdot y^{n}$ \\
6. & $f(z ; n, 1, \ldots, 1)=\int_{z}^{1} \frac{(x-z)^{k-1}}{(k-1) !} x^{n-1} f(x) d x$ & $P(y) \cdot y^{n+k-1}$ \\
7. & $-\frac{d f(x)}{d x}$ & $P(y) \cdot y^{n_{1}+n_{2}+\ldots+n_{k}}$ \\
8. & $\left(-\frac{d}{d x}\right)^{k}\left[x^{n} f(x)\right]$ & $P(y) y^{n-k}$ \\
\hline
\end{tabular}

Table 2: Main results of CMM generalization of the DGLAP equations. The first column contains functions which are the generalized CMM (2.2) and the second column contains their corresponding DGLAP evolution kernels.

one obtains the relation between "ordinary" $(n+1)$ th cut moments and weighted $n$th CMM (2.11):

$$
\int_{x_{0}}^{1} x^{n} f(x) d x=x_{0} \sum_{k=0}^{\infty} \int_{x_{0}}^{1} \frac{\ln ^{k}\left(x / x_{0}\right)}{k !} f(x) x^{n} \frac{d x}{x}
$$

where all terms of the equation evolve with the same DGLAP kernel $P(y) \cdot y^{n+1}$.

In Fig. 2, we show the the generalized CMM for $n=0$,

$$
I_{k}\left(x_{0}\right) \equiv \int_{x_{0}}^{1} \frac{\ln ^{k}\left(x / x_{0}\right)}{k !} g_{1}^{N S}(x) \frac{d x}{x}=\left(\frac{\ln ^{k}(1 / t)}{k !} * g_{1}^{N S}\right)\left(x_{0}\right),
$$

multiplied by $x_{0}$, as a function of $k$ for different cut points $x_{0}$. We plot also partial sums $x_{0} \sum_{j=0}^{k} I_{j}\left(x_{0}\right)$ which, according to (3.3), in the large limit $k \approx 20$ give the contribution to the Bjorken sum rule:

$$
\int_{x_{0}}^{1} g_{1}^{N S}(x) d x=x_{0} \sum_{k=0}^{\infty} I_{k}\left(x_{0}\right) .
$$

Taking into account that experimental data from different laboratories are obtained with different uncertainties, particularly in the small- $x$ region, it seems to be useful to consider besides the "usual" contributions to the Bjorken sum rule, their generalizations (3.1). This will allow for a reasonable 


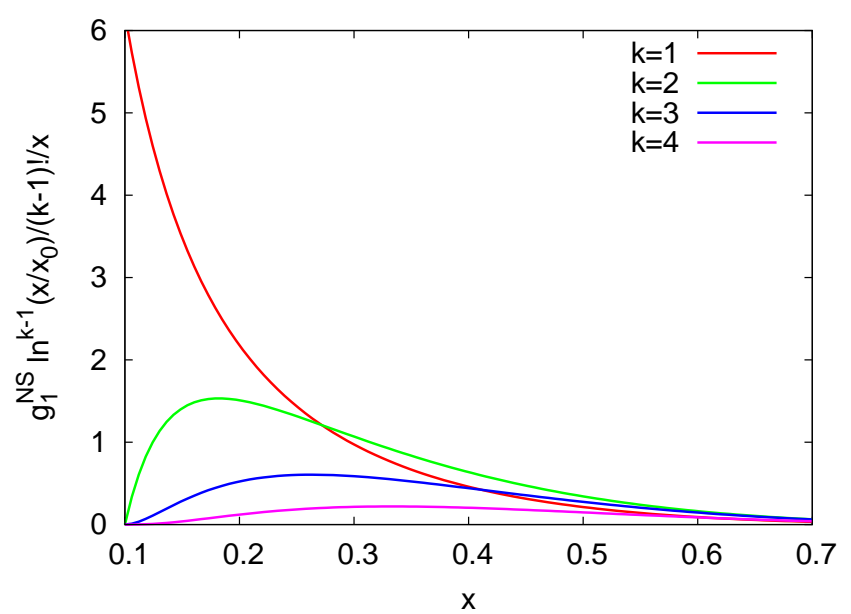

Figure 1: The integrands of the generalized Bjorken sum rule (3.1) for different $k$ and Regge type input parameterizations of $g_{1}^{N S}\left(x, Q_{0}^{2}\right): \sim(1-x)^{3}$ and $x_{0}=0.1$.

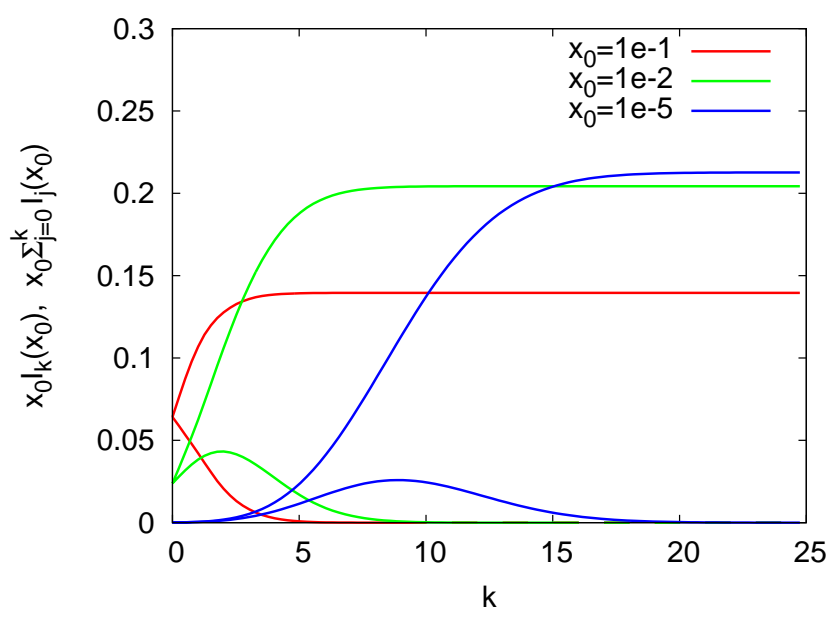

Figure 2: Generalized CMM (3.4), multiplied by $x_{0}$, versus $k$ at different cut points $x_{0}$ (lower plots). The upper plots represent partial sums $x_{0} \sum_{j=0}^{k} I_{j}\left(x_{0}\right)$, respectively.

comparison of the data as contributions from the smaller Bjorken $x$ region suffering from larger experimental errors to enter in this generalized Bjorken sum rule with essentially smaller weights. Let us finally notice that using the property of convolution

$$
\int_{0}^{1} x^{n-1} d x \int_{x}^{1} \frac{d y}{y} f(y) g\left(\frac{x}{y}\right)=\left[\int_{0}^{1} x^{n-1} f(x) d x\right] \times\left[\int_{0}^{1} x^{n-1} g(x) d x\right]
$$

and the integral representation of factorial

$$
n !=\int_{0}^{1} \ln ^{n}(1 / x) d x
$$

one can easily obtain that for each $k$ the first moment of the generalized CMM $I_{k}(3.4)$ is the same 
and equal to the usual BSR:

$$
\int_{0}^{1} I_{k}(x) d x \equiv \int_{0}^{1}\left(\frac{\ln ^{k}(1 / t)}{k !} * g_{1}^{N S}\right)(x) d x=\int_{0}^{1} g_{1}^{N S}\left(x, Q^{2}\right) d x=\mathrm{BSR} .
$$

The same property (3.8) as well as the same DGLAP evolution remains valid for more general conditions. Indeed, for any normalized weight $\omega(t)$ the $\mathrm{CMM} G_{\omega}$, presented as a Mellin convolution of $g_{1}^{N S}$ and $\omega$,

$$
\begin{aligned}
G_{\omega}\left(x, Q^{2}\right)=\left(\omega * g_{1}^{N S}\right)(x) \equiv & \int_{x}^{1} \omega(x / z) g_{1}^{N S}\left(z, Q^{2}\right) \frac{d z}{z}, \\
& \int_{0}^{1} \omega(t) d t=1,
\end{aligned}
$$

is normalized as $g_{1}$,

$$
\int_{0}^{1} G_{\omega}\left(x, Q^{2}\right) d x=\int_{0}^{1} g_{1}^{N S}\left(x, Q^{2}\right) d x=\mathrm{BSR} .
$$

The corresponding DGLAP kernel $P_{\omega}$ for $G_{\omega}$ can be obtained directly, $P_{\omega}=\omega * P * \omega^{-1}=P$
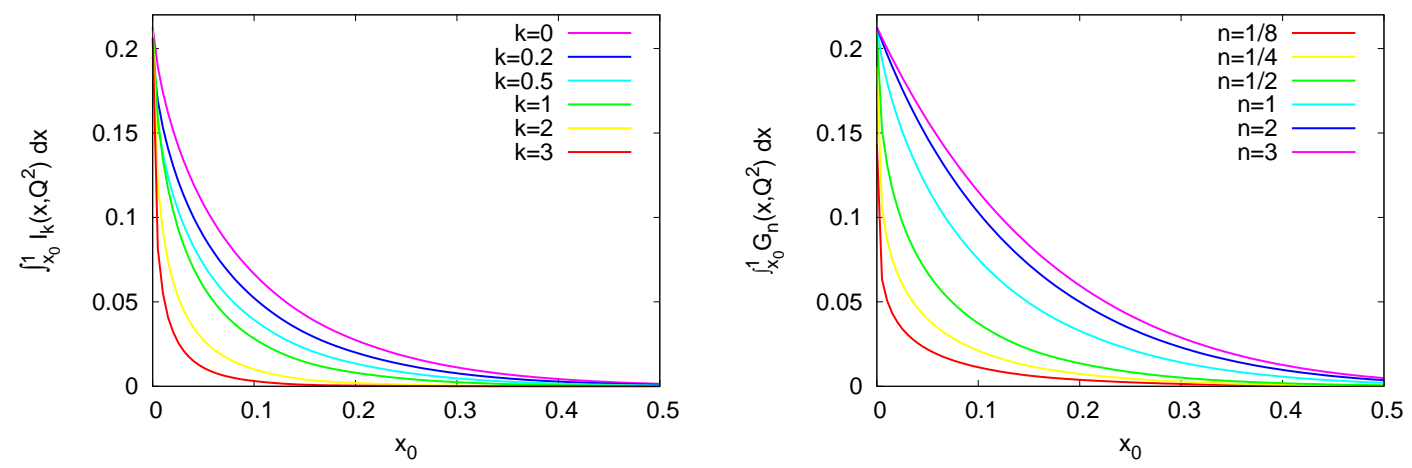

Figure 3: The cut first moments of the generalized CMM $I_{k}$ (3.4) and $G_{n}$ (3.9), where $\omega=n t^{n-1}$, for different $k$ and $n$ versus the cut point $x_{0}$. In the limit $x_{0} \rightarrow 0$ all curves go to the BSR, according to (3.8) and (3.11).

in virtue of the properties of Mellin convolution ${ }^{1}$. Therefore, $I_{k}$ and $G_{\omega}$ have the same evolution DGLAP kernel $P(y)$, as $g_{1}^{N S}$ has. Hence, the corresponding cut first moments of $I_{k}$ and $G_{\omega}$ go to the BSR limit as the cut point $x_{0}$ goes to zero. It is tempting to fit such $\omega$ for $G_{\omega}$ to approach this limit in the smoothest way. This very smooth behaviour of the integral near $x_{0}=0$ allows one to estimate the value of the BSR from the cut integrals $\int_{x_{0}}^{1} G_{\omega}\left(x, Q^{2}\right) d x$ at $x_{0} \neq 0$. The attempts are shown in Fig. 3 for the case $\omega(t)=n t^{n-1}$ and also can be tested experimentally.

More detailed analysis of the recent experimental data with the use of the generalized CMM will be presented in the forthcoming paper [18].

\section{Conclusions}

We reviewed the main results on the generalized CMM approach. General CMM (multiple integrations as well as multiple differentiations of the original parton distribution) obey the same

\footnotetext{
${ }^{1}$ notation $\omega^{-1}$ means that $\left(\omega * \omega^{-1}\right)(x)=\left(\omega^{-1} * \omega\right)(x)=\delta(1-x)$
} 
DGLAP evolution equations with simply modified evolution kernel. As experiments provide data for cut moments, the main advantage of CMMA is that fundamental properties of the nucleon can be studied in a experimentally restricted range of Bjorken- $x$. In this way, one can avoid uncertainties from the unmeasurable regions. The CMM approach is a novel tool providing a rich variety of further possible ways to test QCD. The choice of suitable classes of CMM for the available experimental kinematic range enables enhancement of the $x$-region with smaller uncertainties. For instance, we propose to consider besides the contributions to the Bjorken sum rule their generalizations that will allow for reasonable comparison of theoretical and experimental data obtained in different laboratories.

\section{References}

[1] V. N. Gribov and L. N. Lipatov, Deep inelastic ep scattering in perturbation theory, Sov. J. Nucl. Phys. 15 (1972) 438.

[2] V. N. Gribov and L. N. Lipatov, $e+e-$ pair annihilation and deep inelastic ep scattering in perturbation theory, Sov. J. Nucl. Phys. 15 (1972) 675.

[3] Yu. L. Dokshitzer, Calculation of the structure functions for deep inelastic scattering and e $+e-$ annihilation by perturbation theory in quantum chromodynamics, Sov. Phys. JETP 46 (1977) 641.

[4] G. Altarelli and G. Parisi, Asymptotic freedom in parton language, Nucl. Phys. B 126 (1977) 298.

[5] S. Forte and L. Magnea, Truncated moments of parton distributions, Phys. Lett. B 448 (1999) 295 [hep-ph/9812479].

[6] S. Forte, L. Magnea, A. Piccione and G. Ridolfi, Evolution of truncated moments of singlet parton distributions, Nucl. Phys. B 594 (2001) 46 [hep-ph / 0006273$].$

[7] A. Piccione, Solving the Altarelli-Parisi equations with truncated moments, Phys. Lett. B 518 (2001) 207 [hep-ph / 0107108$].$

[8] S. Forte, J. Latorre and L. Magnea and A. Piccione, Determination of $\alpha_{s}$ from scaling violations of truncated moments of structure functions, Nucl. Phys. B 643 (2002) 477 [hep-ph/ 0205286$].$

[9] D. Kotlorz and A. Kotlorz, Evolution equations for truncated moments of the parton distributions, Phys. Lett. B 644 (2007) 284 [hep-ph / 0610282$].$

[10] D. Kotlorz and A. Kotlorz, Evolution equations of the truncated moments of the parton densities. A possible application, Acta Phys. Pol. B 40 (2009) 1661 [arXiv: 0906.0879 ].

[11] D. Kotlorz and A. Kotlorz, Truncated Mellin moments: Useful relations and implications for the spin structure function g2, Acta Phys. Pol. B 42 (2011) 1231 [arXiv: 1106 . 3753].

[12] D. Kotlorz and A. Kotlorz, Evolution of the truncated Mellin moments of the parton distributions in QCD analysis, Phys. Part. Nucl. Lett. 11 (2014) 357 [arXiv: 1405.5315$].$

[13] A. Psaker, W. Melnitchouk, M. E. Christy and C. Keppel, Quark-hadron duality and truncated moments of nucleon structure functions, Phys. Rev. C 78 (2008) 025206 [arXiv: 0803.2055 ].

[14] D. Kotlorz and S.V. Mikhailov, Cut moments and a generalization of DGLAP equations, JHEP 06 (2014) 065 [arXiv: 1404.5172 ].

[15] O. Teryaev, QCD evolution and density matrix positivity, in Proceedings of the XXX PNPI International Winter School, February 2005, Repino, Russia, edited by V. Kudryavtsev and L. Lipatov, pp. 90-102, Gatchina, PNPI, 2007. 
[16] X. Artru, M. Elchikh, Jean-Marc Richard, J. Soffer and O. Teryaev, Spin observables and spin structure functions: inequalities and dynamics, Phys.Rept. 470 (2009) 1 [arXiv: 0802.0164 ].

[17] E. G. Floratos, R. Lacaze and C. Kounnas, Space and timelike cut vertices in QCD Beyond the Leading Order. 1. Nonsinglet Sector, Phys. Lett. B 98 (1981) 89; ibid. 285.

[18] D. Kotlorz and S.V. Mikhailov, Recent results on the generalization of DGLAP evolution equations in Cut Mellin Moments approach, in preparation. 